\title{
The association between personal technology use and cognition: Does use matter?
}

\author{
Daniel A. Harris $\mathrm{MPH}^{\mathrm{a}, *}$, Kali S. Thomas $\mathrm{PhD}^{\mathrm{b}, \mathrm{c}}$, Gary Epstein-Lubow $\mathrm{MD}^{\mathrm{c}, \mathrm{d}, \mathrm{e}}$, Eric \\ Jutkowitz $\mathrm{PhD}^{\mathrm{b}, \mathrm{c}}$ \\ ${ }^{a}$ Division of Epidemiology, Dalla Lana School of Public Health, University of Toronto, To- \\ ronto, ON, CA; ${ }^{b}$ Center of Innovation in Long-term Services and Supports, Department \\ of Veterans Affairs Medical Center, Providence, RI, USA; ${ }^{\circ}$ Center for Gerontology and \\ Healthcare Research, School of Public Health, Brown University, Providence, RI, USA;

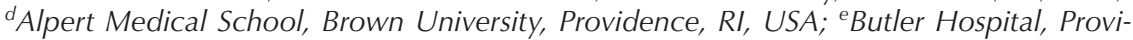 \\ dence, RI, USA; *Corresponding author: daniel.harris@mail.utoronto.ca
}

\begin{abstract}
Background: Population-based assessments of the association between personal technology use and cognitive performance have shown positive effects among seniors. However, prior cognitive function, which predicts future technology use, is seldom considered as a potential confounder in previous cross-sectional studies.

Objective: Estimate the association between two measures of personal technology use (emailing/texting and computer internet use for administrative tasks) and cognitive performance among a population-based sample of older adults, while adjusting for prior cognitive function.

Methods: Data were obtained from the 2016 and 2017 National Health and Aging Trends Study. Among 3,695 older adults, we measured the association between emailing/texting and computer internet use for administrative tasks and delayed and immediate word recall, and clock draw score. Survey-weighted multivariable linear regression estimated these relationships before and after adjusting for demographic factors, socioeconomic factors, physical and mental health, and prior cognitive function.

Results: Participants who never emailed/texted remembered $0.39(95 \% \mathrm{CL}=-0.54,-0.24)$ fewer words for delayed word recall, $0.49(95 \% \mathrm{CL}=-0.64,-0.35)$ fewer words for immediate word recall, and had $0.19(95 \% \mathrm{CL}=-0.29,-0.09)$ lower clock draw scores compared to participants who emailed/texted most days. Participants without computer access remembered $0.28(95 \% \mathrm{CL}=-0.47,-0.08)$ fewer words for delayed word recall and 0.44 $(95 \% \mathrm{CL}=-0.58,-0.30)$ fewer words for immediate word recall compared to users of 4 or more Internet functions.

Conclusion: We found significant differences in delayed and immediate word recall, and clock draw scores between the highest and lowest users of emailing/texting. Greater internet use was significantly associated with higher delayed and immediate recall scores compared to participants without computer access.
\end{abstract}

Keywords: Cognition, memory, text messaging, electronic mail, cell phone

\section{INTRODUCTION}

By 2050 , the number of adults $\geq 65$ years of age is projected to more than double from 41.1 million to 83.7 million (Ortman, Velkoff, \& Hogan, 2014). Despite historically low rates of computer/ mobile phone use among older adults, evidence indicates that seniors now account for the most rapid growth of technology users (Hart, Chaparro, \& Halcomb, 2008; Newburger, 2001). Although it remains unclear if the observed increases in technology use are due to the aging of higheruse groups and/or general increases in use, the growing prevalence of technology use among older adults has led researchers to explore the potential effects of personal technology (e.g., cell phones) on cognitive health (Elliot, Mooney, Douthit, \& Lynch, 2014; Kane et al., 2017).
Previous research on technology use, cognition, and aging has been informed by the "Use it or Lose it" and "Cognitive Reserve" frameworks (Hultsch, Hertzog, Small, \& Dixon, 1999; Salthouse, 1991; Scarmeas \& Stern, 2003). In brief, these theoretical frameworks suggest that greater engagement in intellectual, social, and physical activities throughout life is associated with slower age-related cognitive decline. A growing body of research is attempting to identify if the use of personal technology is stimulating enough to slow age-related cognitive decline.

In one randomized trial, older adults who received a computerized brain-training program had significantly higher cognitive performance scores at 6 months follow-up compared to con- 
trol participants (Miller et al., 2013). Similar findings have been replicated in other randomized trials (Basak, Boot, Voss, \& Kramer, 2008; Djabelkhir et al., 2017; also see, O'Shea, De Wit, \& Smith, 2017). In addition to being associated with better cognitive performance on behavioral tasks, a recent neuroimaging study revealed that less daily computer use, assessed by minutes of use per day over one month, was associated with smaller hippocampal volume among cognitively intact older adults (Silbert et al., 2016). However, experimental studies evaluate carefully designed interventions and can include non-representative samples. Therefore, there is a need to conduct population-based assessments of the association between everyday personal technology use and cognition to expand the generalizability of results.

Several observational studies have found that technology use is associated with better cognitive performance in older adults (Elliot et al., 2014; Fazeli, Ross, Vance, \& Ball, 2013; though see, Salthouse, Berish, \& Miles, 2002). Using a sample of community-dwelling older adults in the United States, one study cross-sectionally found that mobile phone use was associated with better cognitive performance (Elliot et al., 2014). A second cross-sectional study showed that computer experience was associated with better performance across multiple cognitive measures (Fazeli et al., 2013). However, as with all cross-sectional designs, it remains unclear whether personal technology use confers cognitive benefits or if cognitively robust individuals engage with technology more than their cognitively impaired counterparts. In fact, Slegers et al.'s (2009) randomized control trial found that internet training had little effects on cognitive performance and that participants with better verbal memory and inhibitory control used their computers more often compared to participants with poorer performance.

To control for qualities of cognition that may lead to greater technology use, Tun \& Lachman (2010) cross-sectionally examined the association between the frequency of computer use and a battery of cognitive tests, while controlling for a composite measure of basic cognitive ability. Their results showed that participants who used a computer daily had modestly higher scores on the Brief Test of Adult Cognition by Telephone (Tun \& Lachman, 2006; Tun \& Lachman, 2005) assessment compared to participants who used the computer once per month. The authors also reported that computer use was associated with better executive function even when controlling for basic cognitive ability. To date, Tun et al. (2010) remains one of the few studies to use a population-based sample of adults to investigate how technology use is associated with cognitive health while adjusting for basic cognitive ability.
The current study examines the association between two facets of personal technology use (emailing/texting and computer internet use for administrative tasks) and cognitive performance, while controlling for cognitive performance in the previous year. Our study extends the literature by stratifying personal technology use by two different tasks (emailing/texting and computer internet use for administrative tasks [e.g., online banking]), as each process is theoretically related to cognitive performance via different causal mechanisms (Barnes, Mendes de Leon, Wilson, Bienias, \& Evans, 2004; Seeman, Lusignolo, Albert, \& Berkman, 2001). Additionally, we more directly control for confounding by considering cognitive performance in the previous year. Last, compared to Tun et al. (2012), who included a sample of 635 older adults, our study includes a large population-based sample of 3,695 adults 65 years and older to more robustly examine this relationship in older age.

\section{Methods \\ Data source}

Data were obtained from the 2016 and 2017 rounds of National Health and Aging Trends Study (NHATS; www.NHATS.org). The NHATS is a population-based prospective cohort of older adults that began in 2011. The present study used data from participants in $2017(n=6,312)$ and their associated cognitive performance in 2016.

As residents of nursing homes are a distinct population, we restricted the current analysis to participants living in the community $(n=5,177)$. Among community-dwelling participants, we excluded participants with a proxy interview $(n=201)$, participants whoever had a dementia diagnosis $(n=239)$, and participants with invalid data for the independent variables, dependent variables, or covariates $(n=982)$. The final analytical sample consisted of 3,695 participants.

The authors' IRB ruled the present study was exempt from the institution IRB ethics approval because these data have already been collected, are non-identifiable, and are publicly available.

\section{Dependent variables}

Episodic memory and executive function were assessed in 2016 and 2017 using the Consortium to Establish a Registry for Alzheimer's Disease (CERAD) immediate and delayed recall tests and clock drawing test (Morris et al., 1989). To test episodic memory, participants were presented with a list of 10 words and asked to recall them immediately (i.e., immediate word recall) and after a five-minute delay (i.e., delayed word recall). Scores for both the immediate and delayed word recall tests ranged from 0 to 10 , represented by the total number of correctly remembered 
words. As a test of executive function and visuospatial abilities, participants were given 120 seconds to draw an analog clock representing "10 after 11". Trained research staff scored drawings from 0 ("not recognizable as a clock") to 5 ("accurate depiction of a clock") (Wolf-Klein, Silverstone, Levy, \& Brod, 1989). Scores in 2017 represent the primary dependent variables and the corresponding 2016 scores were included as covariates to control for prior cognitive function.

\section{Independent variables}

Similar to Elliot et al. (2014), we created two ordinal scales representing facets of personal technology use: emailing/texting and computer internet use for administrative tasks. Greater social engagement, irrespective of technology use, is associated with better cognitive performance among older adults (Barnes et al., 2004; Seeman et al., 2001). Therefore, we conceptualized emailing/texting, which is a social function, as theoretically distinct from computer internet use for administrative tasks. The NHATS measures participants' emailing/texting behavior and their use of various computer internet functions for administrative tasks. Participants were first asked if they emailed or texted in the last month (yes or no). Participants reporting "yes" were then asked, "In the last month, how often did you send messages by email or texting? Would you say, most days, some days or rarely?". Participants who reported to not email or text in the first question were added to create a fourth category representing no use ("never"). Final response categories for emailing/ texting use included "most days", "some days", "rarely", and "never". Computer internet use was categorized into a four-point ordinal scale: $0=$ does not have a working computer/has a computer but does not know how to use it/does not access a computer from a second location; $1=$ has a working computer but does not use the internet; 2 = has a working computer and uses the internet for at most 3 functions; 3 = has a working computer and uses the internet for 4 or more functions (Elliot et al., 2014). The NHATS internet functions included grocery shopping, banking, refilling prescriptions, contacting medical providers, managing/researching medical insurance, and researching health conditions.

\section{Covariates}

Several sociodemographic characteristics were included as confounders (Elliot et al., 2014; Fazeli et al., 2013). Sex, age (65-79, 70-74, 75-79, 8084, 85-90, and 90+), education (less than high school, high school/vocational training/some college, and college or higher education), race/ ethnicity (White (non-Hispanic), Black (non-Hispanic), Hispanic, and Other (two or more race/ ethnicities, Pacific Islander, Asian, American Indian)) were included in the analysis. We also included NHATS' first imputed yearly income in 2017, but excluded 2 participants with annual incomes above the $99^{\text {th }}$ percentile.

\section{Physical health}

Physical health status has been shown to be associated with both the independent and dependent variables of interest (Fratiglioni, Paillard-Borg, \& Winblad, 2004; Kesse-Guyot et al., 2012). Similar to Elliot et al., (2014), we included participants' subjective self-rated health (excellent, very good, good, fair, and poor) as a covariate.

\section{Mental health and social connection}

Depressive symptoms were measured using the Patient Health Questionnaire-2 (PHQ-2), which has been previously validated to detect clinically significant symptoms of depression in geriatric populations (Li, Friedman, Conwell, \& Fiscella, 2007). Participants were asked, "Over the last month, how often have you (1) had little interest or pleasure in doing things, and (2) felt down, depressed, or hopeless?" Valid response categories included, not at all, several days, more than half the days, nearly every day. Responses to each question ranged from 0 (not at all) to 3 ('nearly every day) and were summed to obtain a composite measure of depressive symptoms that ranged from 0 to 6 .

We controlled for social connection by including a term for the number of individuals a respondent indicated they could talk to about important things (between 0 and 5). Finally, we controlled for participants' marital status in 2017 (married/ living with a partner, separated/divorced, widowed, or never married).

\section{Data analysis}

We described the analytical sample and compared participants across the levels of emailing/ texting and computer internet use. Similar to prior studies using the NHATS cognitive performance measures, multivariable linear regression was used to estimate unadjusted and adjusted associations between each cognitive performance dependent variable (immediate word recall, delayed word recall, and clock draw score) and each ordinal measure of emailing/texting and computer internet use separately (Wennberg, Hagen, Gottesman, Zipunnikov, Kaufmann et al., 2017; Wennberg, Gottesman, Kaufmann, Albert, Chen-Edinboro et al., 2014).

All analyses used 2017 NHATS survey weights to account for the complex survey design. All analyses were conducted in Stata 15.1 (Statacorp, 2017).

\section{Results}

A total of 3,695 participants met the study's inclusion criteria, representing 26,913,733 older adults living in the contiguous United States. 
Table 1. 2017 National Health and Aging Trends Study (NHATS) participants' characteristics, by emailing/texting use $(N=3,695)$.

\begin{tabular}{|c|c|c|c|c|c|}
\hline & \multicolumn{4}{|c|}{ Emailing/Texting use } & \multirow[b]{2}{*}{$p$} \\
\hline & $\begin{array}{l}\text { Most days } \\
(n=1,231)\end{array}$ & $\begin{array}{c}\text { Some days } \\
(n=574)\end{array}$ & $\begin{array}{c}\text { Rarely } \\
(\mathrm{n}=329)\end{array}$ & $\begin{array}{c}\text { Never } \\
(n=1,561)\end{array}$ & \\
\hline \multicolumn{6}{|l|}{ Dependent variables of interest $^{1}$} \\
\hline 2017 Delayed word recall, mean (SE) & $4.80(0.06)$ & $4.27(0.09)$ & $4.01(0.14)$ & $3.09(0.05)$ & $<0.001$ \\
\hline 2017 Immediate word recall, mean (SE) & $6.00(0.05)$ & $5.43(0.07)$ & $5.25(0.08)$ & $4.40(0.04)$ & $<0.001$ \\
\hline 2017 Clock draw score, mean (SE) & $4.27(0.04)$ & $4.13(0.04)$ & $4.08(0.07)$ & $3.67(0.04)$ & $<0.001$ \\
\hline \multicolumn{5}{|l|}{ Sex, n (\%) } & $<0.001$ \\
\hline Female & $736(59.88)$ & $304(51.02)$ & $173(50.66)$ & $879(51.93)$ & \\
\hline Male & $495(40.12)$ & $270(48.98)$ & $156(49.34)$ & $682(48.07)$ & \\
\hline \multicolumn{5}{|l|}{ Race/Ethnicity, n (\%) } & $<0.001$ \\
\hline White & $1014(88.40)$ & $470(85.95)$ & $247(82.59)$ & $916(69.67)$ & \\
\hline Black & $149(4.67)$ & $69(4.84)$ & $62(7.33)$ & $474(13.9)$ & \\
\hline Other race/more than one & $34(3.55)$ & $17(5.10)$ & $4(1.28)$ & $34(3.71)$ & \\
\hline Hispanic & $34(3.38)$ & $18(4.10)$ & $16(8.80)$ & $137(12.71)$ & \\
\hline \multicolumn{5}{|l|}{ Age, n (\%) } & $<0.001$ \\
\hline $65-69$ & $170(24.23)$ & $48(15.88)$ & $22(15.07)$ & $70(9.79)$ & \\
\hline $70-74$ & $506(46.04)$ & $184(41.41)$ & $93(39.57)$ & $274(25.87)$ & \\
\hline $75-79$ & $309(18.67)$ & $142(21.92)$ & $105(26.25)$ & $362(24.56)$ & \\
\hline $80-84$ & $160(8.00)$ & $109(13.42)$ & $54(10.77)$ & $393(20.08)$ & \\
\hline $85-90$ & $70(2.59)$ & $67(5.29)$ & $37(5.56)$ & $298(13.61)$ & \\
\hline $90+$ & $16(0.46)$ & $24(2.08)$ & $18(2.78)$ & $164(6.09)$ & \\
\hline \multicolumn{5}{|l|}{ Education, n (\%) } & $<0.001$ \\
\hline Less than high school & $50(4.07)$ & $32(5.83)$ & $26(8.04)$ & $513(31.47)$ & \\
\hline High school/Vocational school & $495(39.89)$ & $284(51.23)$ & $190(55.87)$ & $828(55.54)$ & \\
\hline College + & $686(56.04)$ & $258(42.94)$ & $113(36.09)$ & $220(12.99)$ & \\
\hline \multicolumn{5}{|l|}{ Marital status, n (\%) } & $<0.001$ \\
\hline Married/Partnered & $729(62.39)$ & $363(66.98)$ & $178(60.86)$ & $628(45.83)$ & \\
\hline Separated/Divorced & $197(16.32)$ & $71(12.50)$ & $41(10.26)$ & $230(15.17)$ & \\
\hline Widowed & $274(19.30)$ & $126(17.65)$ & $99(24.54)$ & $625(34.37)$ & \\
\hline Never married & $31(1.99)$ & $14(2.87)$ & $11(4.35)$ & $78(4.63)$ & \\
\hline \multicolumn{5}{|l|}{ Self-rated health, n (\%) } & $<0.001$ \\
\hline Excellent & $212(19.35)$ & $62(11.98)$ & $26(8.80)$ & $96(7.17)$ & \\
\hline Very good & $483(40.85)$ & $208(36.66)$ & $112(30.19)$ & $368(22.94)$ & \\
\hline Good & $419(31.06)$ & $218(37.53)$ & $118(36.65)$ & $606(38.47)$ & \\
\hline Fair & $94(6.99)$ & $76(12.18)$ & $60(18.28)$ & $398(24.65)$ & \\
\hline Poor & $23(1.75)$ & $10(1.66)$ & $13(6.08)$ & $93(6.77)$ & \\
\hline Social network size ${ }^{2}$, mean (SE) & $2.65(0.05)$ & $2.30(0.07)$ & $2.15(0.11)$ & $1.97(0.04)$ & $<0.001$ \\
\hline Depressive symptoms ${ }^{3}$, mean (SE) & $0.54(0.04)$ & $0.77(0.06)$ & $0.77(0.09)$ & $1.11(0.05)$ & $<0.001$ \\
\hline Income, mean (SE) & $\begin{array}{l}99,395.01 \\
(6,012.24) \\
\end{array}$ & $\begin{array}{l}73,618.81 \\
(6,414.38) \\
\end{array}$ & $\begin{array}{c}73,906.59 \\
(12,231.34) \\
\end{array}$ & $\begin{array}{l}40,270.24 \\
(2,308.87) \\
\end{array}$ & $<0.001$ \\
\hline 2016 Delayed word recall ${ }^{4}$, mean (SE) & $4.99(0.07)$ & $4.32(0.10)$ & $4.20(0.13)$ & $3.17(0.06)$ & $<0.001$ \\
\hline 2016 Immediate word recall ${ }^{4}$, mean (SE) & $6.05(0.05)$ & $5.50(0.06)$ & $5.37(0.10)$ & $4.54(0.04)$ & $<0.001$ \\
\hline 2016 Clock draw score ${ }^{4}$, mean (SE) & $4.18(0.03)$ & $4.13(0.05)$ & $4.11(0.07)$ & $3.69(0.03)$ & $<0.001$ \\
\hline \multicolumn{6}{|c|}{$\begin{array}{l}\text { Notes: } \mathrm{SE}=\text { standard error } \\
\text { 1. Delayed word recall and immediate word recall score represent the number of correctly remembered words and ranges from } 0 \text { to } 10 \text {. Clock draw score represents the } \\
\text { accuracy of a hand-drawn clock and ranges from } 0 \text { to } 5 \text {, with } 5 \text { representing a fully accurate depiction. All dependent variables were assessed in } 2017 . \\
\text { 2. Social network size reflects a self-reported number social connections and ranges from } 0 \text { to } 5 \text {. } \\
\text { 3. Depressive symptoms were assessed via the PHQ-2 questionnaire and ranges from } 0 \text { to } 6 \text { depressive symptoms. } \\
\text { 4. Delayed word recall and immediate word recall score represent the number of correctly remembered words and ranges from } 0 \text { to } 10 \text {. Clock draw score represents the } \\
\text { accuracy of a hand-drawn clock and ranges from } 0 \text { to } 5 \text {, with } 5 \text { representing a fully accurate depiction. All of these measures were assessed in } 2016 \text {. } \\
\text { 5. Unweighted } \mathrm{Ns} \text { and survey weighted means and proportions are reported. }\end{array}$} \\
\hline
\end{tabular}

In the 30 days prior to participants' 2017 assessment, 1,231 (42.67\%) emailed/texted most days, $574(17.09 \%)$ emailed/texted some days, $329(8.64 \%)$ emailed/texted rarely, and 1,561 (31.61\%) never emailed/texted. Participants who emailed/texted most days had significantly greater proportions of women, White race/ethnicity, younger age, higher educational attainment, and better self-rated health. All three cognitive performance scores in 2017 were also significantly higher with the increasing use of emailing/texting. Additionally, participants who emailed/ texted more frequently in 2017 had higher mean cognitive performance scores in 2016. Cognitive performance scores in 2017 were significantly correlated with scores in 2016, with medium to large effect sizes (Tables $1 \& 2$ ).

In the 30 days before the 2017 assessment, 588 $(20.59 \%)$ participants used 4 or more computer internet functions, 1,212 (39.16\%) participants used at most 3 internet functions, 755 (16.21\%) participants had access to a computer but no internet and 1,140 (24.04\%) had no computer access. Observed differences in participant characteristics were similar when stratified by com- 
Table 2. Survey-weighted correlation coefficients between 2016 and 2017 National Health and Aging Trends Study (NHATS) participants' cognitive performance scores $(N=3,695)$.

\begin{tabular}{lccc}
\hline & $\begin{array}{c}\text { 2017 Delayed word } \\
\text { recall }(\mathbf{0 - 1 0})\end{array}$ & $\begin{array}{c}\text { 2017 Immediate word } \\
\text { recall }(\mathbf{0 - 1 0})\end{array}$ & $\begin{array}{c}\text { 2017 Clock draw } \\
\text { score }(\mathbf{0 - 5})\end{array}$ \\
\hline $\mathbf{2 0 1 6}$ Delayed word recall $(\mathbf{0}-\mathbf{1 0})$ & $0.565^{* * *}$ & -- & -- \\
$\mathbf{2 0 1 6}$ Immediate word recall $(\mathbf{0 - 1 0})$ & -- & $0.570^{* * *}$ & -- \\
$\mathbf{2 0 1 6}$ Clock draw score $(\mathbf{0 - 5})$ & -- & -- & $0.392^{* * *}$ \\
\hline
\end{tabular}

puter internet use to emailing/texting use (data not shown).

\section{Emailing/texting}

After adjusting for confounding, we found significant differences in cognitive performance between never emailing/texting and emailing/texting most days (Table 3). Compared to participants who emailed/texted most days, those who never emailed/texted remembered $0.39(95 \% \mathrm{CL}=-0.54$, -0.24) fewer words on the delayed word recall test, $0.49(95 \% \mathrm{CL}=-0.64,-0.35)$ fewer words on the immediate word recall test, and had $0.19(95 \% \mathrm{CL}$ $=-0.29,-0.09)$ lower scores on the clock draw test. Adjusted mean predicted scores for each dependent variable are reported in Table 4.
There were no significant differences between adjacent emailing/texting use categories for delayed word recall or clock draw scores. However, participants who emailed/texted most days had significantly higher immediate word recall scores compared to participants who emailed/texted some days $(p=0.012)$. There was no significant difference between participants who emailed/ texted some days compared to rarely $(p=0.599)$, and participants who rarely emailed/texted had significantly higher scores than those who never emailed/texed $(\mathrm{p}=0.012)$ (Figure 1).

Computer internet use for administrative tasks After adjusting for confounding, we found significantly lower delayed and immediate word recall scores among participants without computer access (delayed word recall: $\beta=-0.28,95 \% \mathrm{CL}=-$ $0.47,-0.08$; immediate word recall: $\beta=-0.44$,

\section{A. Emailing/Texting Use}
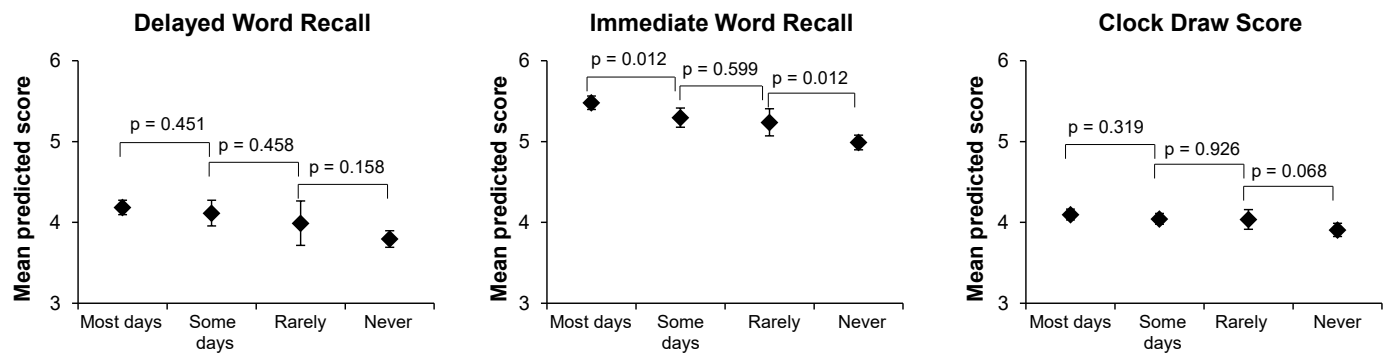

Levels of emailing/texting

\section{B. Computer Internet Use for Administrative Tasks}


\section{Levels of computer use}

Figure 1. Adjusted mean predicted cognitive performance scores by levels of personal technology use. Note: A. Emailing/Texting Use represents the adjusted mean predicted delayed word recall. immediate word recall, and clock draw score across levels of emailing and texting. B. Computer Internet Use for Administrative Tasks represents the adjusted mean predicted delayed word recall, immediate word recall, and clock draw score across levels of computer use. Means were predicted after each linear regression model and are adjusted for age, sex, race/ethnicity, income, education, social network size, marital status, depressive symptoms, self-rated health, and the associated cognitive performance score in $2016.95 \%$ confidence intervals are plotted for each estimate. 


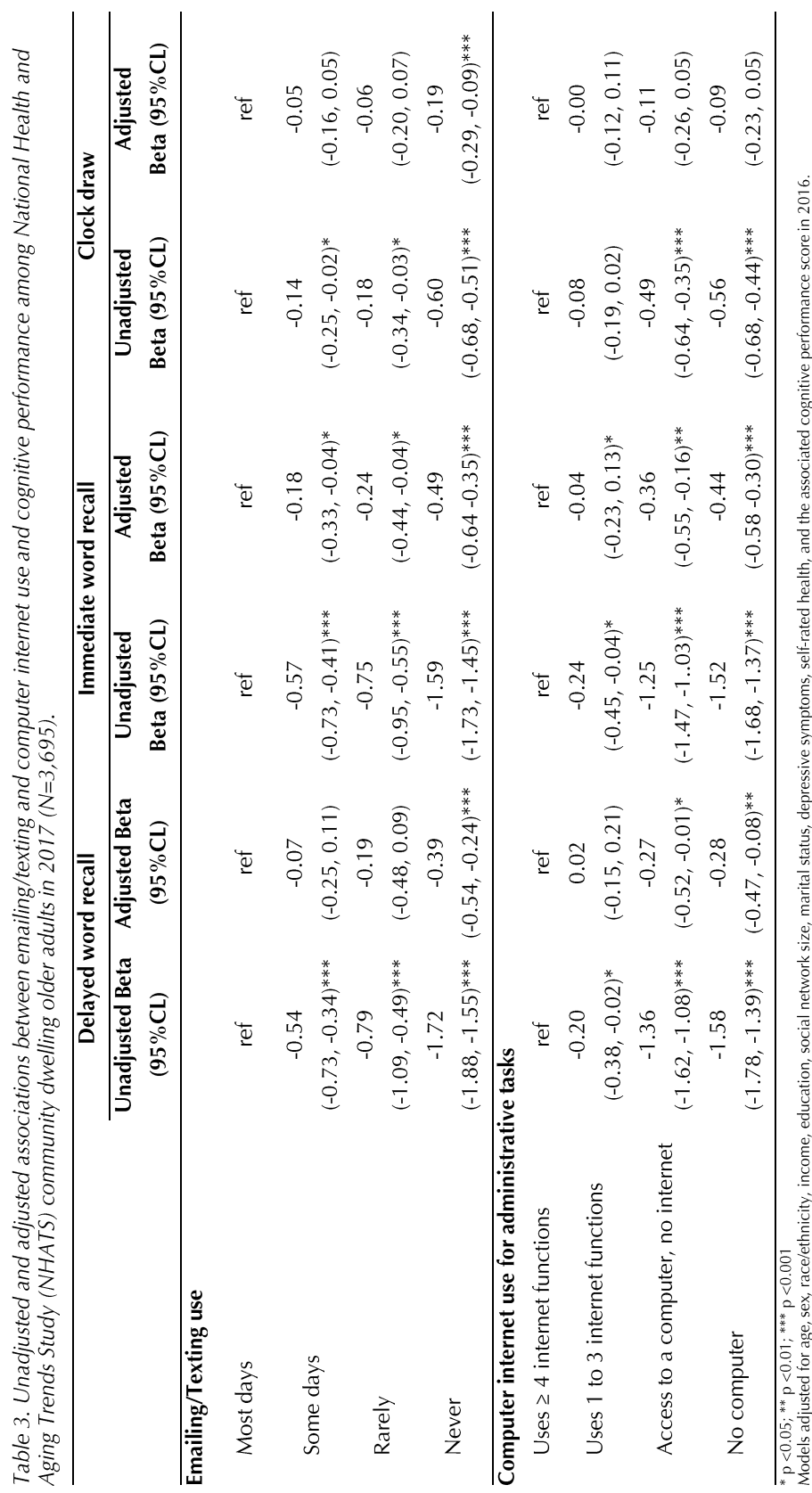

were found between participants with computer access but no internet and participants with no computer access across all cognitive performance scores. However, participants who had computers but no internet access had significantly lower delayed word recall $(p=0.005)$, immediate word recall $(p<0.001)$, and clock draw $(\mathrm{p}=0.034)$ scores compared to participants who used 3 or fewer internet functions (Figure 1).

\section{Discussion}

We examined the association between emailing/texting and computer internet use for administrative tasks and cognitive performance among a populationbased sample of older adults living in the contiguous United States. After controlling for confounding, we found significant differences in delayed word recall, immediate word recall, and clock draw scores between those that emailed/texted most frequently and least frequently. Similarly, participants who used the internet for administrative tasks had significantly higher delayed word recall and immediate recall scores compared to participants who did not use the internet for administrative tasks.

Our findings echo previous population-based cross-sectional studies that also found significant positive associations between personal technology use and cognitive performance (Elliot et al., 2014; Fazeli et al., 2013; Tun \& Lachman, 2010). When comparing the extremes of emailing/texting (i.e., emailing/texting most days to never) and computer internet use (i.e., uses 4+ internet functions to no computer access), our findings suggest that these two facets of personal technology use are associated with better cognitive performance among older adults. We observed insignificant differences between most adjacent emailing/texting and computer internet use categories (with one exception being immediate word recall for emailing/texting use). Experimental studies with greater control of the independent variable will be needed to elucidate whether a dose-response relationship between emailing/texting behaviors and computer internet performance scores. No significant differences 
Table 4. Adjusted mean predicted scores of cognitive performance for levels of emailing/texting and computer internet use among National Health and Aging Trends Study (NHATS) community dwelling older adults in 2017 (N=3,695).

\begin{tabular}{|c|c|c|c|}
\hline & $\begin{array}{c}\text { Delayed word recall } \\
\text { Mean }(95 \% \mathrm{CL})\end{array}$ & $\begin{array}{l}\text { Immediate word recall } \\
\text { Mean }(95 \% \mathrm{CL})\end{array}$ & $\begin{array}{l}\text { Clock draw score } \\
\text { Mean }(95 \% \mathrm{CL})\end{array}$ \\
\hline \multicolumn{4}{|l|}{ Emailing/Texting use } \\
\hline Most days & $4.18(4.09,4.27)$ & $5.48(5.40,5.56)$ & $4.10(4.03,4.16)$ \\
\hline Some days & $4.11(3.95,4.27)$ & $5.30(5.18,5.42)$ & $4.04(3.98,4.11)$ \\
\hline Rarely & $3.99(3.71,4.26)$ & $5.24(5.07,5.40)$ & $4.04(3.91,4.16)$ \\
\hline Never & $3.79(3.69,3.90)$ & $4.99(4.90,5.08)$ & $3.91(3.83,3.99)$ \\
\hline \multicolumn{4}{|c|}{ Computer internet use for administrative tasks } \\
\hline Uses $\geq 4$ internet functions & $4.03(3.89,4.18)$ & $5.38(5.26,5.50)$ & $4.02(3.92,4.12)$ \\
\hline Uses 1 to 3 internet functions & $4.06(3.96,4.17)$ & $5.34(5.24,5.44)$ & $4.02(3.96,4.07)$ \\
\hline Access to a computer, no internet & $3.77(3.60,3.94)$ & $5.02(4.90,5.14)$ & $3.91(3.82,4.01)$ \\
\hline No computer & $3.76(3.65,3.88)$ & $4.94(4.86,5.02)$ & $3.93(3.85,4.01)$ \\
\hline
\end{tabular}

Models adjusted for age, sex, race/ethnicity, income, education, social network size, marital status, depressive symptoms, self-rated health, and the associated cognitive performance score in 2016.

use and cognitive performance exists.

Controlling for cognitive performance in the previous year is a strength of the current study, and in part, likely explains the small effect size. Conceptualized as a confounder, cognitive performance in 2016 is likely causally associated with the independent and dependent variables of interest but does not temporally mediate the relationship. Previous cross-sectional studies that have not considered prior performance can introduce bias because as Slegers et al. (2009) found, participants with better cognitive performance engage with technology more often compared to participants with poorer cognitive performance.

While the cross-sectional association between emailing/texting and computer internet use on cognitive performance is modest in effect size, we hypothesize that the observed differences will increase over time. This hypothesis is supported by the slow nature of cognitive change. One prospective cohort study of community-dwelling cognitively intact older adults found insignificant differences in the rate of cognitive decline over 3 years of follow-up but found significant evidence of cognitive decline after 6 years of follow-up (Small, Fratiglioni, Viitanen, Winblad, \& Backman, 2000). Therefore, a logical next step is to explore how these facets of everyday technology use are associated with cognitive performance over several years of follow-up.

Our findings extend the results of previous studies, but we remain cautious to interpret their clinical significance. For example, although statistically significant, we found that participants who never emailed/texted remembered only 0.39 fewer words on delayed word recall and 0.49 fewer words on immediate word recall compared to participants who emailed/ texted most days after adjusting for confounding. Recent work by Goldstein and colleagues (2018) evaluated the incremental validity of the Montreal Cognitive Assessment (MoCA) and compared differences in MoCA scores across participants with Alzheimer disease, mild cognitive impairment, and normal cognition. The Cohen's d comparing effect sizes on the language and visuospatial subtests between cognitively normal participants and participants with mild cognitive impairment were small (0.29 and 0.28, respectively). As the authors noted, differentiating between participants with normal cognitive abilities and mild cognitive impairment can be distinguished by subtle differences in performance. While the cognitive assessments used in our study differ from Goldstein et al. (2018), our observed differences of 0.39 on delayed word recall and or 0.49 on immediate word recall between emailing/texting most days compared to never, might suggest the difference between normal cognition and mild cognitive impairment in some patients; however, additional work is required to assess the clinical significance.

The present study has several limitations. First, although we excluded participants with dementia, the potential for recall bias remains for the selfreported independent variables, and covariate measures. Second, emailing/texting and computer internet use for administrative tasks captures only two facets of personal technology use, and each element was grouped into four broad categories. Third, other forms of personal technology, such tablets, and other handheld devices were not measured. Fourth, there is a potential for differential misclassification of the independent variables, such that individuals with better cognitive performance more accurately report their technology use behaviors compared to participants with poorer cognitive health; however, the direc- 
tion of this potential bias is challenging to predict. Last, and related to the prior limitation, there is likely heterogeneity within personal technology use categories thereby potentially violating the consistency condition (Hernán \& Robins, 2018).

Despite these limitations, our study adds to the literature by directly considering prior cognitive performance in our assessment of current technology use behaviors and cognitive performance. Additionally, our measures of cognitive performance were objectively measured using clinically validated measures. Future studies may longitudinally explore this relationship and/ or estimate the direct effects of technology use and the joint effects of technology use and so- cial connection on cognitive performance. As with previous studies, the observed association between emailing/texting use and cognitive performance may be attributed to, in part, elements of social connection.

\section{Conclusions}

Our results indicated that emailing/texting and computer internet use for administrative tasks (e.g., online banking) were modestly associated with better scores on delayed and immediate word recall, and clock draw scores. These findings suggest that everyday personal technology use may have a small protective benefit on older adults' cognitive performance; which has implications in seniors' quality of life and social well-being.

\section{Acknowledgements}

The authors report no potential or actual conflicts of interest. The NHATS is sponsored by the National Institute on Aging (grant number NIA U01AG032947) through a cooperative agreement with the Johns Hopkins Bloomberg School of Public Health. The views expressed in this article are those of the authors and do not necessarily reflect the position or policy of the Department of Veterans Affairs or the United States government.

\section{Funding}

- Kali Thomas is supported by a Career Development Award from the Veterans Health Administration (CDA14-422).

- Eric Jutkowiz is supported by grants from the National Institute on Aging (1R21AG059623-01 and 1R01AG060871-01). - Gary Epstein-Lubow has received support from Hebrew SeniorLife, Butler Hospital, Alpert Medical School of Brown University, NIH, Administration for Community Living, The John A. Hartford Foundation, The Rhode Island Foundation, and the Health and Aging Policy Fellows Program. Dr. Epstein-Lubow is a non-federal member of the U.S. Dept. of Health and Human Service's Advisory Council for Alzheimer's Research, Care and Services; the views and opinions expressed in this manuscript are his own and do not represent views of the Council.

\section{References}

Barnes, L. L., Mendes de Leon, C. F., Wilson, R. S., Bienias, J. L., \& Evans, D. A. (2004). Social resources and cognitive decline in a population of older African Americans and whites. Neurology, 63(12), 2322-2326.

Basak, C., Boot, W. R., Voss, M. W., \& Kramer, A. F. (2008). Can training in a real-time strategy video game attenuate cognitive decline in older adults? Psychology and Aging, 23(4), 765-777. https://doi. org/10.1037/a0013494

Djabelkhir, L., Wu, Y. H., Vidal, J. S., Cristancho-Lacroix, V., Marlats, F., Lenoir, H., Rigaud, A. S. (2017). Computerized cognitive stimulation and engagement programs in older adults with mild cognitive impairment: comparing feasibility, acceptability, and cognitive and psychosocial effects. Clinical Interventions in Aging, 12, 1967-1975. https://doi. org/10.2147/CIA.S145769

Elliot, A. J., Mooney, C. J., Douthit, K. Z., \& Lynch, M.
F. (2014). Predictors of older adults' technology use and its relationship to depressive symptoms and well-being. Journals of Gerontology, Series B: Psychological Sciences and Social Sciences, 69(5), 667-677. https://doi.org/10.1093/geronb/gbt109

Fazeli, P. L., Ross, L. A., Vance, D. E., \& Ball, K. (2013). The relationship between computer experience and computerized cognitive test performance among older adults. Journals of Gerontology, Series B: Psychological Sciences and Social Sciences, 68(3), 337346. https://doi.org/10.1093/geronb/gbs071

Fratiglioni, L., Paillard-Borg, S., \& Winblad, B. (2004). An active and socially integrated lifestyle in late life might protect against dementia. Lancet Neurology, 3(6), 343-353. https://doi.org/10.1016/S14744422(04)00767-7

Goldstein, F. C., Milloy, A., Loring, D. W., \& for the Alzheimer's Disease Neuroimaging, I. (2018). Incremental Validity of Montreal Cognitive Assessment Index Scores in Mild Cognitive Impairment and Alzheimer Disease. Dementia and Geriatric Cognitive Disorders, 45(1-2), 49-55. https://doi. org/10.1159/000487131

Hart, T., Chaparro, B., \& Halcomb, C. (2008). Evaluating websites for older adults: Adherence to seniorfriendly guidelines and end-user performance. Behaviour \& Information Technology, 27(3), 191-199.

Hernán, M. A. Robins, J. M. (2018). Causal Inference. Boca Raton, FL: Chapman \& Hall/CRC, forthcoming.

Hultsch, D. F., Hertzog, C., Small, B. J., \& Dixon, R. A. (1999). Use it or lose it: engaged lifestyle as a buffer of cognitive decline in aging? Psychology and Aging, 14(2), 245-263.

Kane, R. L., Butler, M., Fink, H. A., Brasure, M., Davila, H., Desai, P., Barclay, T. (2017) Interventions to Prevent Age-Related Cognitive Decline, Mild Cognitive Impairment, and Clinical Alzheimer's-Type Dementia; Agency for Healthcare Research and Quality (US): Rockville, MD.

Kesse-Guyot, E., Charreire, H., Andreeva, V. A., Touvier, M., Hercberg, S., Galan, P., \& Oppert, J. M. (2012). Cross-sectional and longitudinal associations of different sedentary behaviors with cognitive performance in older adults. PLoS One, 7(10), e47831. https://doi.org/10.1371/journal.pone.0047831 
Li, C., Friedman, B., Conwell, Y., \& Fiscella, K. (2007). Validity of the Patient Health Questionnaire 2 (PHQ-2) in identifying major depression in older people. Journal of the American Geriatrics Society, 55(4), 596-602. https://doi.org/10.1111/j.15325415.2007.01103.x

Miller, K. J., Dye, R. V., Kim, J., Jennings, J. L., O'Toole, E., Wong, J., \& Siddarth, P. (2013). Effect of a computerized brain exercise program on cognitive performance in older adults. The American Journal of Geriatric Psychiatry, 21(7), 655-663. https://doi. org/10.1016/j.jagp.2013.01.077

Morris, J. C., Heyman, A., Mohs, R. C., Hughes, J. P., van Belle, G., Fillenbaum, G., Clark, C. (1989). The Consortium to Establish a Registry for Alzheimer's Disease (CERAD). Part I. Clinical and neuropsychological assessment of Alzheimer's disease. Neurology, 39(9), 1159-1165.

Newburger, E. C. (2001). Home Computers and Internet Use in the United States: August 2000. U.S. Census Bureau, Washington, DC. Report retrieved from the World Wide Web at http://www.census.gov/ prod/2001pubs/p23-207.pdf.

O'Shea, D. M., De Wit, L., \& Smith, G. E. (2017). Doctor, Should I Use Computer Games to Prevent Dementia? Clinical Gerontologist, 1-14. https://doi.org /10.1080/07317115.2017.1370057

Ortman, J. M., Velkoff V. A, Hogan, H. (2014). An Aging Nation: The Older Population in the United States, Current Population Reports, Current Population Reports, P25-1140. US Census Bureau, Washington, DC.

Salthouse, T. A. (1991). Theoretical perspectives on cognitive aging. Hillsdale, N.J.: L. Erlbaum Associates.

Salthouse, T. A., Berish, D. E., \& Miles, J. D. (2002). The role of cognitive stimulation on the relations between age and cognitive functioning. Psychology and Aging, 17(4), 548-557.

Scarmeas, N., \& Stern, Y. (2003). Cognitive reserve and lifestyle. Journal of Clinical and Experimental Neuropsychology, 25(5), 625-633. https://doi. org/10.1076/jcen.25.5.625.14576

Seeman, T. E., Lusignolo, T. M., Albert, M., \& Berkman, L. (2001). Social relationships, social support, and patterns of cognitive aging in healthy, high-func- tioning older adults: MacArthur studies of successful aging. Health Psychology, 20(4), 243-255.

Silbert, L. C., Dodge, H. H., Lahna, D., Promjunyakul, N. O., Austin, D., Mattek, N., Kaye, J. A. (2016). Less Daily Computer Use is Related to Smaller Hippocampal Volumes in Cognitively Intact Elderly. Journal of Alzheimers Disease, 52(2), 713-717. https://doi.org/10.3233/JAD-160079

Small, B. J., Fratiglioni, L., Viitanen, M., Winblad, B., \& Backman, L. (2000). The course of cognitive impairment in preclinical Alzheimer disease: threeand 6-year follow-up of a population-based sample. JAMA Neurology, 57(6), 839-844.

Statacorp. (2017). Stata Statistical Software: Release 15. College Station, TX: StataCorp, LLC.

Tun, P. A., \& Lachman, M. E. (2006). Telephone assessment of cognitive function in adulthood: the Brief Test of Adult Cognition by Telephone. Age and Ageing, 35(6), 629-632. https://doi.org/10.1093/ageing/afl095

Tun, P. A., \& Lachman, M. E. (2010). The association between computer use and cognition across adulthood: use it so you won't lose it? Psychology and Aging, 25(3), 560-568. https://doi.org/10.1037/a0019543

Tun, P. A., \& Lachman, M.E. (2005). The Brief Test of Adult Cognition by Telephone (BTACT) Technical Report. Retrieved from Brandeis University.

Wennberg, A. M., Gottesman, R. F., Kaufmann, C. N., Albert, M. S., Chen-Edinboro, L. P., Rebok, G. W., Spira, A. P. (2014). Diabetes and cognitive outcomes in a nationally representative sample: The National Health and Aging Trends Study. International Psychogeriatrics, 26(10), 1729-1735. https:// doi.org/10.1017/s1041610214001380

Wennberg, A. M. V., Hagen, C. E., Gottesman, R. F., Zipunnikov, V., Kaufmann, C. N., Albert, M. S., Spira, A. P. (2017). Longitudinal association between diabetes and cognitive decline: The National Health and Aging Trends Study. Arch Gerontol Geriatr, 72, 39-44. https://doi.org/10.1016/j.archger.2017.05.005

Wolf-Klein, G. P., Silverstone, F. A., Levy, A. P., \& Brod, M. S. (1989). Screening for Alzheimer's disease by clock drawing. Journal of the American Geriatrics Society, 37(8), 730-734. 